\title{
Detection of Phospholipase A2 Receptor Related Membranous Nephropathy in Membranous Nephropathy Patient
}

\section{Alaa Elmaghraby ${ }^{a}$, Wesam M Ismail $^{\mathrm{b}}$, Amira Ahmedabdelnaby ${ }^{\mathrm{a}}$, Ali Taha Ali ${ }^{\mathrm{c}}$, Eman Muhammed Salaheldeen $^{\mathrm{a}}$}

${ }^{\text {a }}$ Pathology Department,Faculty of Medicine, SohagUniversity,Sohag,Egypt.

${ }^{\mathrm{b}}$ PathologyDepartment,Faculty of Medicine, Beni-SuefUniversity, Beni-Suef,Egypt.

${ }^{\mathrm{c}}$ Nephrology Department, Sohag University Hospital, SohagUniversity,Sohag,Egypt.

\begin{abstract}
Background: Membranous glomerulonephritis (MGN) considered as the most common causes of nephrotic syndrome (NS) in the world. Eighty percent of cases are classified as primary MGN. Primary MGN is an autoimmune disease in which autoantibodies against podocyte antigens forming subepithelial immune deposits and result in NS. Autoantibodies against M-type phospholipase A2 receptor (PLA2R) were found in $70-80 \%$ of patients with primary MGN but not in those with secondary MGN or other renal diseases. So, we aim to identify PLA2R associated primary MGN.

Patient and methods: Out of 5000 native biopsies received from 2014-17, 600 were diagnosed as MGN, out of which 62 were stained for anti-PLA2R by immunoperoxidase. Nine cases of Lupus (SLE) Class V, 12 of hepatitis C\&B Virus MGN (10 HCV+ve and $2 \mathrm{HBV}+\mathrm{ve}$ ) were used as controls. Positive was determined as diffuse glomerular capillary wall staining.Results: Anti-PLA2R was positive in $61 \%$ $(38 / 62)$ of cases (33 idiopathic cases and 5 cases of the control group3:HCV, $1 \mathrm{HBV}, 1 \mathrm{SLE})$. None of the clinical parameters showed significance difference but nephrotic range proteinuria in the anti-PLA2R +ve group was more (66\% vs 46\%). Histologically, onlymesangial matrix expansion was significantly different in the aPLA2R -ve group (33\% vs $10 \%, \mathrm{p}=0.04)$.

Conclusion: anti-PLA2R tissue staining is a reliable and specific method to identify primary MGN and should be done routinely in all MGN cases even those identified as secondary on clinical basis.

Key words:Membernous glomerulonephritis, anti-PLA2R, nephrotic syndrome.
\end{abstract}

\section{Introduction}

Membranous glomerulonephritis is the most common cause of nephrotic syndrome in adults. Two forms of MGN have classically been described: primary form and secondary form of MGN which represent $80 \%$ and $20 \%$ of cases, respectively (Pozdzik et al.,2018).

Histologically, it is characterized by subepithelial immune deposits, with subsequent thickening of the basement membrane, without cellular proliferation and infiltration. The immune deposits appear as granular deposits of $\operatorname{IgG}$ with immunofluorescence or immunoperoxidase on light microscopy, or as electron-dense deposits on electron microscopy (Kao et al., 2016).

Primary MGN is related to autoimmune disorders caused by the presence of circulating antibodies against native podocyte antigens such as NEP, PLA2R1, and thrombospondin type 1 domain containg $7^{\mathrm{a}}$ (THSD7A) or by antibodies developed against external antigens such as cationic bovine serum albumin (Pozdzik etal.,2018).

Autoantibodies against PLA2R were found in 70$80 \%$ of patients with primary MGN (Debiec\& Ronco, 2011).

Assessment of PLA2R antigen in biopsy specimens is more sensitive than serological test 
alone for diagnosis of PLA2R-related MGN (Svobodova et al., 2012).

Secondary MGN is associated with autoimmune diseases e.g., systemic lupus erythematosus, infections e.g., hepatitis B or hepatitis C, drugs e.g., penicillamine, gold \& NSAIDs, and malignancies e.g., colon or lung cancer (Svobodova et al., 2012).

\section{Patients and methods \\ Case selection}

Five thousand cases of native renal biopsy (nontransplanted kidney) received from 2013 to 2017 in Path Lab of Dr Wesam Ismail, 600 cases were diagnosed as MGN.

Sixty two cases included in this study, 41 cases were suggested to be primary MGN; without known secondary causes of MGN, and 21 cases suggested to be secondary MGN; 9 cases with SLE; 12 cases of hepatitis B or C.

All specimens were formalin fixed and paraffinembedded and tissue sections were examined histopathologically by routine H\&E, PAS and Masson trichrome stains to evaluate their diagnosis.

\section{Inclusion criteria}

- Adequate tissue sample with at least 10 glomeruli.

- Enough residual tissues in the paraffin blocks.

- Available clinical data.

- Adult patient (above 18 years old).

\section{Exclusion criteria}

- Cases with a membranous pattern, which proved to be non-membranous glomerulonephritis

by immunohistochemistry (IHC) eg; chronic thrombotic microangiopathy, early diabetic changes or Alport's disease).

\section{Immunohistochemical staining(IHC)}

PLA2R1 mouse monoclonal antibody (clone no., CL0474, Abcam, Atlas Antibody, Sweden; product number (AMAb90772)was used in 1/1000 dilution. IHC staining with PLA2R antibody revealed faint positivity in normal kidneys. Anti PLA2R was selectively expressed on podocytes in a fine granular pattern.

The immune deposits are localized in the subepithelial space in the same location of $\mathrm{IgG}$ immunoglobulins staining (Beck et al., 2009).

Statistical Analysis
All patients had been analyzed using Statistical package for Social Sciences (SPSS-16).P value less than 0.05 was considered statistically significant.

\section{Results}

MGN represent 12\% (600/5000) from the collected native biopsies. By staining specimens with $\mathrm{H} \& \mathrm{E}$, PAS and Masson trichrome; we demonstrated the diffuse global thickening of GBM in all studied cases.

The age range of the studded patients was 18-76 years, and the mean age was $41.5 \pm 14$ year. The male to female ratio was nearly $1: 1$. Mean serum creatinine at time of biopsy was $1.08 \pm 1.06 \mathrm{mg} / \mathrm{dl}$. Impaired serum creatinine (above $1.2 \mathrm{mg} / \mathrm{dl}$ ) was seen in 17 cases (27\%). As regard to proteinuria, there were $36(58 \%)$ cases with nephrotic range proteinuria (more than $3.5 \mathrm{~g} / 24$ hours), and 26 $(52 \%)$ cases with non-nephrotic range proteinuria as shown in table (1).

\begin{tabular}{|l|l|l|}
\hline \multicolumn{2}{|c|}{} & Value \\
\hline Age (years) & Mean \pm SD & $41.5 \pm 14$ \\
\cline { 2 - 3 } & Range & $18-76$ \\
\hline \multirow{2}{*}{ Sex } & Male No. (\%) & $30(49 \%)$ \\
\cline { 2 - 3 } & Female No. (\%) & $32(51 \%)$ \\
\hline \multirow{2}{*}{$\begin{array}{l}\text { Serum } \\
\text { creatinine } \\
\text { (mg/dl) }\end{array}$} & Mean \pm SD & $1.1 \pm 1$ \\
\cline { 2 - 3 } $\begin{array}{l}\text { Proteinuria } \\
(24 \text { h mean }\end{array}$ & Range & $0.3-6$ \\
\cline { 2 - 3 } $\begin{array}{l}\text { urinary } \\
\text { protein in } \\
\text { gm) }\end{array}$ & Inpaired creatinine & $17(27 \%)$ \\
\cline { 2 - 3 } & Range SD & $4.5 \pm 4.7$ \\
\cline { 2 - 3 } & $\begin{array}{l}\text { Nophrotic range } \\
\text { range nephrotic }\end{array}$ & $36-25$ \\
\hline
\end{tabular}

Table (1) Demographic and basic clinical data of the study group

There was no significant difference between the aPLA2R positive and negative cases among all clinical data (age, sex, impaired serum creatinine andproteinuria). We found that, although nephrotic proteinuria was much higher among aPLA2R positive cases $(66 \%)$ compared to aPLA2R negative cases (46\%); this difference was not statistically significant. Also aPLA2R positive cases had higher risk for creatinine impairment (32\%) compared to aPLA2R negative cases $(21 \%)$ without statistically significant difference. 
From the data sheets of the collected specimens all 62 studied cases were positive for IgG immunostaining, while $26(42 \%)$ cases were positive for $\mathrm{C} 3$ immunostaining. Only 42 cases were immunostained with $\mathrm{C} 4 \mathrm{~d}$ and all of them $(100 \%)$ were positive. All cases in the current study were stained with aPLA2R but only $38(61 \%)$ cases were positivefigure (1).

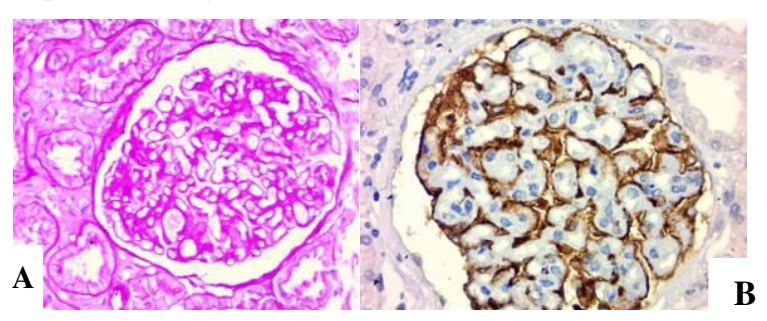

Figure (1): A) H\&E, a glomerulus show thick glomerular basement membrane. B) IHC staining by aPLA2R, a glomerulus from patient with idiopathic MGN. The subepithelial deposits are diffuse strongly positive for aPLA2R (x400).

Anti PLA2R antibody were positive in 38/62 cases $(61 \%)$ and negative in $24 / 62$ cases (39\%).

Of the studied cases $9 / 62$ clinically suggestive to be SLE (secondary MGN), 8 were PLA2R negative which represent $8 / 24$ (33\%) of the PLA2R negative cases. Only one/38 (3\%) aPLA2R positive case was diagnosed as SLE. This difference was statistically significant $(\mathrm{p}=0.001)$. And among aPLA2R negative cases there are $8 / 24(33 \%)$ cases had hepatitis (B or C), compared to 4/38 (10\%) cases of aPLA2R positive. The difference was statistically significant $(\mathrm{p}=0.046)$ (Table 2$)$.

Table (2): Relation between known causes of 2ry MGN and positivity of aPLA2R

\begin{tabular}{|l|l|l|l|l|}
\hline \multirow{2}{*}{} & \multicolumn{2}{l|}{ aPLA2R } & \multirow{2}{*}{ Total } \\
\cline { 2 - 4 } & \multicolumn{2}{l|}{ Positive } & Negative & T \\
\hline SLE & No & $1(3 \%)$ & $8(33 \%)$ & 9 \\
\hline Hepatitis & No & $4(10 \%)$ & $8(33 \%)$ & 12 \\
\hline Total & No & $38(100 \%)$ & $24100 \%)$ & 62 \\
\hline
\end{tabular}

Chi square $=11.174, \mathrm{p}$ value $=0.001(\mathrm{~S})$ for SLE

Chi square $=4.902, p$ value $=0.046(\mathrm{~S})$ for Hepatitis

Mesangial proliferation was significantly higher among aPLA2R negative cases 8/24 (33\%) compared to positive cases 4/38 (10\%). The presence of FSGS, GS or Interstitial fibrosis and tubular atrophy showed no significant difference between aPLA2R positive or negative cases.

\section{Discussion}

The prevalence of MGN was $12 \%$ in the current study. It was similar to the prevalence reported in studies of Choi et al. (2001) and Sim et al. (2016) as they found that the prevalence of MGN was about $12.5 \%$. Our finding was higher than that reported by Metwalli et al. (2000) study who recorded 2.5\% MGN among 200 studied cases; this difference may be due to little number of cases in their study.

Regarding the correlation between serum creatinine level and positivity of aPLA2R; we found that serum creatinine was impaired in $12 / 38(32 \%)$ of aPLA2R positive cases compared to only 5/24 (21\%) among aPLA2R negative cases. However, this difference was non-significant. Our results come in agreement with previous studied (Hoxaetal. 2012 \&Sovoboda et al. 2012), who found no significant difference between PLA2R positivity and serum creatinine level. Hihara et al. (2016)found that serum creatinine was slightly lower among aPLA2R positive cases compared to its level in aPLA2R negative cases, but with nonsignificant difference.

Regarding to the comparison between aPLA2R positivity and proteinuria; we found that; although nephrotic proteinuria was much higher among aPLA2R positive cases 25/38 (66\%) compared to that recorded in aPLA2R negative cases $11 / 24$ $(46 \%)$. There was positive correlation between aPLA2R positivity and proteinuria but the difference was statically non-significant. Similar results was recorded by Hihara et al. (2016); who found that proteinuria was much higher among aPLA2R positive cases; which was seen in $55 \%$ of their aPLA2R positive cases compared to only $42 \%$ among aPLA2R negative ones; but with nonsignificant difference. Sovoboda et al. (2012) found also that there was no significant correlation between PLA2R positivity and proteinuria.

In the current study, all MGN biopsies were positive for $\mathrm{IgG}$ and $\mathrm{C} 4 \mathrm{~d}$ with distinct diffuse and global GBM staining. The C4d deposition in GBM of our studied cases, which matched that observed in the GBM of all MGN patients studied by Espinosa-Hernández (2012).

Antiphospholipase A2 receptor antibody was positive in $38 / 62(61 \%)$ cases and negative in $24 / 62$ 
(39\%) cases. This was slightly higher than the prevalence of aPLA2R positive patients in Hihara et al. (2016) study; which was positive in only $52.6 \%$ of their cases. This difference is possibly due to presence of antibodies other than aPLA2R among negative cases or presence of undiagnosed secondary causes.

By observing the correlation between SLE and positivity of aPLA2R our study revealed that, among aPLA2R positive cases, only $1 / 38$ (3\%) case had SLE compared to $8 / 24$ (33\%) cases among aPLA2R negative cases. This difference was statistically significant $(\mathrm{p}=0.001)$. The correlation between aPLA2R positivity and hepatitis (B or C) revealed that, among aPLA2R positive cases, only $4 / 38(10 \%)$ cases had hepatitis, compared to $8 / 24$ $(33 \%)$ among aPLA2R negative cases. This difference was statistically significant $(\mathrm{p}=0.046)$. Sovoboda et al. (2012) found that cases with SLE had no aPLA2R positivity compared to $16 / 37$ $(47 \%)$ cases among aPLA2R negative, with significant difference of this finding. This indicates that aPLA2R antibody mostly not expressed in SLE cases and should be used as a reliable marker to differentiate between primary MGN and secondary MGN.

As regards to associated histopathological findings, we found that mesangial proliferation was lower among aPLA2R positive cases 4/38(10\%) compared to mesangial proliferation in aPLA2R negative cases $8 / 24(33 \%)$. This difference was statistically significant $(\mathrm{p}=046)$. To the best of our knowledge, this pointed was not previously discussed in literature. It needs further studies on a large number of patients to verify it.

There was no significant differences regarding other histological finding (FSGS, GS, Interstitial fibrosis and tubular atrophy, arteriolar hyalinosis and arterial sclerosis) between aPLA2R positive and negative cases. Our results come in agreement with that recorded byHihara et al. (2016); who failed to find any significant relation between aPL2R positivity and associated histopathological renal findings (glomerular sclerosis, interstitial fibrosis and tubular atrophy).

\section{Conclusions}

Anti PLA2R was expressed in most cases with primary MGN. Anti-PLA2R is a reliable method to distinguish between primary and secondary MGN.

\section{References}

Beck Jr, L. H., Bonegio, R. G., Lambeau, G., Beck, D. M., Powell, D. W., Cummins, T. D. \&Salant, D. J. (2009).M-type phospholipase A2 receptor as target antigen in idiopathic membranous nephropathy. $N$ Engl J Med, 361, 11-21.

Choi, I. J., Jeong, H. J., Han, D. S., Lee, J. S., Choi, K. H., Kang, S. W., Ha, S. K., Lee, H. Y. \& Kim, P. K. (2001). An analysis of 4,514 cases of renal biopsy in Korea.

Debiec, H., \&Ronco, P. (2011).PLA2R autoantibodies and PLA2R glomerular deposits in membranous nephropathy. $N$ Engl J Med, 36.

Espinosa-Hernández, M., Ortega-Salas, R., López-Andreu, M., Gómez-Carrasco, J. M., Pérez-Sáez, M. J., Pérez-Seoane, C., \&Aljama-García, P. (2012). C4d as a diagnostic tool in membranous nephropathy. Nefrología (English Edition), 32, 295-299.

Hihara, K., Iyoda, M., Tachibana, S., Iseri, K., Saito, T., Yamamoto, Y., Suzuki, T., Wada, Y., Matsomoto, K. \& Shibata, T. (2016). Anti-phospholipase A2 receptor (PLA2R) antibody and glomerular PLA2R expression in Japanese patients with membranous nephropathy. PLoS One, 11.

Hoxha, E., Kneißler, U., Stege, G., Zahner, G., Thiele, I., Panzer, U., Harendza, S., Helmchen, U. M. \& Stahl, R. A. (2012). Enhanced expression of the M-type phospholipase A2 receptor in glomeruli correlates with serum receptor antibodies in primary membranous nephropathy. Kidney Int, 82, 797-804.

Kao, L., Lam, V., Waldman, M., Glassock, R. J., \& Zhu, Q. (2015). Identification of the immunodominant epitope region in phospholipase A2 receptor-mediating autoantibody binding in idiopathic membranous nephropathy. J Am SocNephrol, 26, 291-301.

Mitwalli, A. H., Al Wakeel, J., Abu-Aisha, H., Alam, A., Al Sohaibani, M., Tarif, 
N., Hammad, D., Askar, A., Sulimani, F., Abid, J. \&Memon, N. (2000). Prevalence of glomerular diseases: King Khalid university hospital, Saudi Arabia. Saudi J Kidney Dis Transplant, 11, 442.

Pozdzik, A., Brochériou, I., David, C., Touzani, F., Goujon, J. M., \&Wissing, K. M. (2018). Membranous Nephropathy and AntiPodocytes Antibodies: Implications for the Diagnostic Workup and Disease Management. Biomed Res Int.

Sim, J. J., Batech, M., Hever, A., Harrison, T. N., Avelar, T., Kanter, M. H., \& Jacobsen, S. J. (2016). Distribution of biopsy-proven presumed primary glomerulonephropathies in 2000-2011 among a racially and ethnically diverse US population. Am J Kidney Dis, 68, 533-544.

Svobodova, B., Honsova, E., Ronco, P., Tesar, V., \&Debiec, H. (2012). Kidney biopsy is a sensitive tool for retrospective diagnosis of PLA2R-related membranous nephropathy. Nephrol Dial Transplant,28, 1839-1844. 